\title{
Neurochemical Effects of Olanzapine in First-Hospitalization Manic Adolescents: A Proton Magnetic Resonance Spectroscopy Study
}

\author{
Melissa P DelBello*,', Kim M Cecil', Caleb M Adler ${ }^{1,3}$, John P Daniels ${ }^{2}$ and Stephen M Strakowski ${ }^{1,3}$ \\ 'Division of Bipolar Disorders Research, Department of Psychiatry, University of Cincinnati College of Medicine, Cincinnati, OH, USA; ${ }^{2}$ mmaging \\ Research Center, Cincinnati Children's Hospital Medical Center, Cincinnati, OH, USA; ${ }^{3}$ Center for Imaging Research, University of Cincinnati \\ College of Medicine, Cincinnati, OH, USA
}

\begin{abstract}
We used proton magnetic resonance spectroscopy ( $\mathrm{H}$ MRS) to compare the in vivo effects of olanzapine on prefrontal $\mathrm{N}$-acetylaspartate (NAA) levels in treatment remitters and nonremitters. Secondary aims of this study were to identify neurochemical predictors of successful olanzapine treatment and other neurochemical effects of olanzapine. In all, 20 adolescents admitted for their first hospitalization for bipolar disorder, type I, manic or mixed and 10 demographically matched healthy subjects were recruited. Manic adolescents were treated with olanzapine monotherapy and scanned at three time points $(N=19)$. Medial and left and right lateral ventral prefrontal NAA, choline, creatine/phosphocreatine, myo-inositol, and glutamate/glutamine were measured at baseline, prior to receiving medication, and on days 7 and 28 of treatment. Healthy subjects did not receive medication but underwent ${ }^{\prime} H$ MRS scans at the same time points to assess for normal variability in metabolites over time. Although there was no overall increase in NAA in manic adolescents following 28 days of treatment with olanzapine, olanzapine remitters $(N=11,58 \%)$ exhibited a greater increase in medial ventral prefrontal NAA compared with nonremitters $(N=8,42 \%, p=0.006)$. Specifically, from baseline to end point, NAA levels decreased in nonremitters $(p=0.03)$ and increased in remitters $(p=0.05)$. Manic adolescents treated with olanzapine had an increase from baseline to day 7 in medial $(p=0.002)$ and right lateral $(p=0.02)$ ventral prefrontal choline. Baseline medial ventral prefrontal choline was greater in olanzapine remitters than in nonremitters $(p=0.001)$. Successful treatment of mania with olanzapine may lead to increased ventral prefrontal neuronal viability and/or function as compared to unsuccessful treatment with olanzapine. Additionally, olanzapine-induced increases in choline may lead to alteration of abnormalities in cell membrane metabolism or second messenger pathways that are thought to be involved in the pathophysiology of bipolar disorder.

Neuropsychopharmacology (2006) 3 I, 1264- 1273. doi: I 0. I038/sj.npp. I 300950; published online 2 November 2005
\end{abstract}

Keywords: magnetic resonance spectroscopy (MRS); bipolar disorder; adolescents; olanzapine

\section{INTRODUCTION}

Although the onset of bipolar disorder commonly occurs during adolescence (Lish et al, 1994), there have been relatively few pharmacological treatment studies involving patients in this age group. As a result, children and adolescents with bipolar disorder must often undergo

This study was presented in part at the American College of Neuropsychopharmacology Annual Meeting, San Juan, Puerto Rico, 2003

* Correspondence: Dr MP DelBello, Division of Bipolar Disorders Research, Department of Psychiatry, University of Cincinnati College of Medicine, 231 Bethesda Avenue, PO Box 670559, Cincinnati, $\mathrm{OH}$ 45267-0559, USA, Tel: + I 513558 5847, Fax: + I 5135583399 , E-mail: delbelmp@email.uc.edu

Received 8 July 2005; revised 7 September 2005; accepted 21 September 2005

Online publication: 27 September 2005 at http://www.acnp.org/ citations/Npp092705050438/default.pdf several unsuccessful medication trials prior to achieving mood stabilization. A recent study found that children and adolescents with bipolar disorder were treated with $3.4 \pm 1.5$ medications and had an average of $6.3 \pm 3.7$ trials of psychotropic medication (Bhangoo et al, 2003). Several open-label and controlled investigations suggest that atypical antipsychotics are effective for the treatment of adolescent mania (Frazier et al, 2001; McConville et al, 2000; DelBello et al, 2002). However, the mechanisms by which these medications exert mood-stabilizing properties remain unknown. Understanding the neurochemical effects and predictors of response to specific pharmacological agents used to treat bipolar disorder would permit targeted treatment interventions so that the optimal mood stabilization can occur for children and adolescents with BP early in their illness course.

Proton magnetic resonance imaging $\left({ }^{1} \mathrm{H}\right.$ MRS) provides in vivo information regarding the concentration of specific biochemicals in localized brain regions. Therefore, ${ }^{1} \mathrm{H}$ MRS 
can be used to identify the neurochemical effects and predictors of response to medications commonly used to treat bipolar disorder. $\mathrm{N}$-acetyl aspartate (NAA) is one neurochemical that is quantified using ${ }^{1} \mathrm{H}$ MRS. NAA is primarily localized to neurons and therefore, decreases in NAA may indicate loss of or impaired neuronal functioning or decreased neuronal viability (Urenjak et al, 1993). Alternatively, NAA is reduced by mitochondrial respiratory chain inhibitors so that decreases in NAA may indicate impaired mitochondrial energy production (Bates et al, 1996). Decreased NAA levels have been reported in the cerebellar vermis (Cecil et al, 2003) and dorsolateral prefrontal cortex (Chang et al, 2003) of unmedicated children and adolescents with bipolar disorder compared with healthy controls.

Recent neuroimaging studies have revealed that lithium may have neurotrophic effects as demonstrated by an increase in cortical NAA and gray matter in bipolar adults following lithium treatment (Moore et al, 2000a,b). In contrast, several studies report decreased or no change in NAA in bipolar patients following treatment with valproate (Silverstone et al, 2003; Cecil et al, 2002). Olanzapine, an atypical antipsychotic, may increase brain-derived neurotrophic factor (BDNF) (Parikh et al, 2004a) and restore loss of cholinergic neurons (Parikh et al, 2003) in rats. Moreover, a recent investigation revealed that exposure to olanzapine leads to increases in nerve growth factor (NGF) in rats, whereas haloperidol decreases, and risperidone does not alter, NGF levels (Parikh et al, 2004b). Together, these data suggest that olanzapine may have neuroprotective and neurotrophic properties. In contrast, another recent study reported a reduction of NAA levels in rat brains following 7 days of olanzapine treatment (Lindquist et al, 2000). However, to our knowledge, the effect of olanzapine therapy on brain NAA in patients with bipolar disorder has not been examined.

With these considerations in mind, the aim of this study was to identify the neurochemcial effects of treatment with olanzapine in first-hospitalization manic adolescents with bipolar disorder. Evaluating first-hospitalization manic adolescents is advantageous in that many of the confounding variables associated with illness chronicity, such as co-occurring substance use disorders and medication exposure, are either absent or present to a lesser extent. We hypothesized that prefrontal NAA would increase following olanzapine treatment, consistent with olanzapine's putative role as a neurotrophic agent. As secondary aims of this study, we compared the effects of successful and unsuccessful treatment with olanzapine on NAA, as well as examined other neurochemical effects and predictors of response to olanzapine.

\section{MATERIALS AND METHODS}

\section{Subjects}

Adolescents admitted for their first hospitalization for bipolar disorder, type I, manic or mixed $(N=20)$ were recruited from consecutive inpatient admissions to the Adolescent Psychiatric Unit at Cincinnati Children's Hospital Medical Center. Patients were included in the study if they were 12-18 years old, met DSM-IV criteria for bipolar disorder, type I, currently mixed or manic, had a Young Mania Rating Scale (YMRS) (Fristad et al, 1992; Young et al, 1978) score of $\geqslant 20$, and had no prior treatment with anticonvulsant, antidepressant, or antipsychotic medications.

Healthy comparison subjects (HC, N=10) between the ages of 12 and 18 years, group-matched for age, education, race, and sex were recruited from the community. The purpose of the HC group was to assess for normal variability in ${ }^{1} \mathrm{H}$ MRS metabolite concentrations between time points. HC adolescents were excluded by a history of psychiatric illness in themselves or any first-degree relative or current treatment with any medications. HC adolescents did not receive medication.

All subjects were excluded by (1) a positive pregnancy test; (2) a history of a substance use disorder within the previous three months or a positive toxicology screen at baseline; (3) a diagnosis of mental retardation (IQ $<70$ ); (4) an unstable medical or neurological disorder; (5) any contraindication to undergoing an MRI scan (such as ferromagnetic implants or claustrophobia); (6) a history of head trauma resulting in a loss of consciousness for more than $5 \mathrm{~min}$; or (7) a Tanner stage of $<3$. Adolescents with bipolar disorder were also excluded by treatment with a benzodiazepine or psychostimulant within $72 \mathrm{~h}$.

Adolescents provided written assent and their parents/ legal guardians provided written informed consent after study procedures were fully explained. This study was approved by the University of Cincinnati and the Cincinnati Children's Hospital Medical Center Institutional Review Boards.

Diagnoses were established using the Washington University at St Louis Kiddie-Schedule for Affective Disorders and Schizophrenia (WASH-U-KSADS) (Geller et al, 2001) by trained child and adolescent psychiatrists with good diagnostic reliability $(\kappa=0.94)$ (DelBello et al, 2002). Adolescents and their parents/legal guardians were interviewed separately. Primary caregiver and child responses were combined in order to ascertain diagnoses. All diagnoses were reviewed in a conference attended by the WASH-U-KSADS interviewer and at least one other child and adolescent psychiatrist and from which a consensus diagnosis was made for each patient. Demographic information was obtained by interviewing the adolescent and their parent/legal guardian. The Self-Rated Tanner Scale was used to assess the stage of adolescent sexual development (Morris, 1980).

\section{Efficacy and Tolerability Measures}

The primary efficacy measure was the YMRS (Fristad et al, 1992; Young et al, 1978). Secondary efficacy measures included the Clinical Global Impression-Bipolar Disorder Version Improvement scores (CGI-BP-I) (National Institute of Mental Health, 1985) ( $1=$ very much improved to $7=$ very much worse) to assess the overall improvement at each visit after olanzapine was initiated. Other secondary efficacy measures included the Clinical Global Assessment Scale (CGAS, $1=$ poor functioning to $100=$ outstanding functioning) (Schaffer et al, 1983) and the Children's Depression Rating Scale-revised (CDRS-R) (Poznanski et al, 1985) to assess overall level of functioning and 
severity of depressive symptoms, respectively. A child and adolescent psychiatrist with previously established reliability for each rating scale (MPD) completed all ratings by interviewing the subject and their primary caregiver $(\mathrm{ICC} \geqslant 0.9)$.

Extrapyramidal symptoms (EPS) were assessed using the Simpson-Angus (Simpson and Angus, 1970), Barnes Akathisia (Barnes, 1989), and Abnormal Involuntary Movement Scales. (AIMS, 1976) Laboratory tests obtained were a complete blood count (CBC), thyroid-stimulating hormone (TSH), liver function tests (LFTs), including alanine aminotransferase (ALT), aspartate aminotransferase (AST), and total bilirubin, and a random glucose level. Vital signs obtained included weight, height, and orthostatic blood pressure and pulse. Electrocardiograms (EKGs) were monitored throughout the study. Additionally, physical examinations were performed on each subject at baseline and end point. Adverse events were assessed at each visit by asking the adolescents and their primary caregiver's open-ended questions about potential side effects.

\section{Study Protocol}

This study was a 4-week open-label prospective study of olanzapine monotherapy. After meeting all inclusion and exclusion criteria, subjects received an initial olanzapine dose of $10 \mathrm{mg}$ at bedtime. Doses were adjusted based on clinical effectiveness and side effects until day 5. A dose range of $10-20 \mathrm{mg} /$ day was permitted. No concomitant medications were permitted at any point during the study. Compliance was measured by pill count at each visit. Additionally, each subject was asked to keep a medication log in order to encourage compliance and identify missed doses. Subjects were discontinued from the study if they missed more than two doses of medication during any 7-day period.

Efficacy and tolerability ratings were performed at baseline and days 7, 14, and 28 or termination from the study. Vital signs were monitored at each visit. Serum monitoring was performed at baseline and end point of study participation.

In-patient attending physicians discharged study participants from the in-patient psychiatry unit when they determined that the subjects were clinically stable (mean \pm SD number of days hospitalized $=6.8 \pm 3.0$ days). All subsequent visits were performed in an outpatient setting.

\section{Proton Magnetic Resonance Spectroscopy (MRS) Procedures}

Subjects underwent an MRS scan at baseline, prior to receiving medication and days 7 and 28 or termination from the study. The scans on days 7 and 28 were performed in order to differentiate between the acute biochemical and antimanic effects of olanzapine, respectively. MRS scans were acquired on a $1.5 \mathrm{~T}$ Signa General Electric MR scanner (Milwaukee, WI).

All subjects (patients and controls) were scanned during the same 13-month period, during which the scanner had the same software and no major upgrades were performed. Additionally, all participants were scanned at approximately the same time in the afternoon to minimize biological variability.

For each subject, a three-plane echo localizer was performed. An axial three-dimensional, inversion recovery prepped, fast spoiled gradient echo (3D IR FSPGR) was also acquired (echo time $(\mathrm{TE})=5.4 \mathrm{~ms}$, repetition time $(\mathrm{TR})=$ $12.5 \mathrm{~ms}$, inversion time $=300 \mathrm{~ms}$, field of view $=24 \mathrm{~cm}$, $1.5-\mathrm{mm}$ thick contiguous slices) in order to provide an anatomic template for MRS voxel placement. Single voxel spectra were prescribed from the axial 3D IR FSPGR series based upon anatomical landmarks. In the event a subject moved or needed to be removed from the scanner, a relocalization imaging sequence was performed. Single voxel magnetic resonance spectroscopy was acquired with the proton brain examination (PROBE) software (General Electric Medical System, Milwaukee, WI) using a point resolved spectroscopy acquisition mode (PRESS) sequence with a TR of $2 \mathrm{~s}$, a TE of $35 \mathrm{~ms}$, and 64 averages and with automatic shimming to achieve water line widths of $<5 \mathrm{~Hz}$. A reference spectrum, acquired within the PROBE acquisition, collected eight acquisitions of unsuppressed water signal for eddy current correction.

Three single voxels, each approximately $8 \mathrm{cc}$ in volume $\left(2 \mathrm{~cm}^{3}\right)$, were positioned in the medial ventral prefrontal cortex (predominately gray matter) and in the left and right ventral lateral prefrontal cortex (predominately white matter). A spectroscopist (KMC), blind to subject diagnosis, positioned the voxels to ensure consistent placement. To minimize variability in voxel relocalization, the position of each subject's head was noted by using the angle of the interpupillary line, which was compared with the transverse landmark light on the scanner. The angle of head flexionextension was determined by moving the subject and table so that the outer canthus and the external auditory meatus were aligned with the transverse landmark light and the position of the intersection with longitudinal light along the midline, typically on the nose. The heads were comfortably secured within the quadrature head coil to minimize head motion.

Each of the three voxels was positioned at the same level to avoid signal artefacts from the orbits. The level was chosen such that the inferior portion of the lateral prefrontal white matter voxels would not include any orbital gyri. The lateral prefrontal white matter voxels were positioned lateral to Brodmann areas 10, 45, and 46. The medial ventral prefrontal (predominately gray matter) voxel included the orbital frontal, middle frontal, and cingulate gyri, which corresponds to Brodmann areas 9, 10, 24, 32, and 47 (Figure 1) (Cecil et al, 2002).

Metabolite concentrations for NAA, choline (Cho), creatine/phosphocreatine (Cr), myo-inositol (mI), and glutamate/glutamine (Glx) in each of the subjects' three spectra were quantitatively measured using a Linear Combination Model of in vitro spectra (LCModel), a commercially available, automatic (user-independent) frequency-domain fitting routine (Provencher, 1993). The method employs a basis set of concentration-calibrated model spectra of individual metabolites to estimate absolute concentrations of similar brain metabolites from in vivo spectral data correcting for residual eddy current effects and actual coil loading by using the transmitter reference amplitude (Provencher, 1993). Each concentration is 

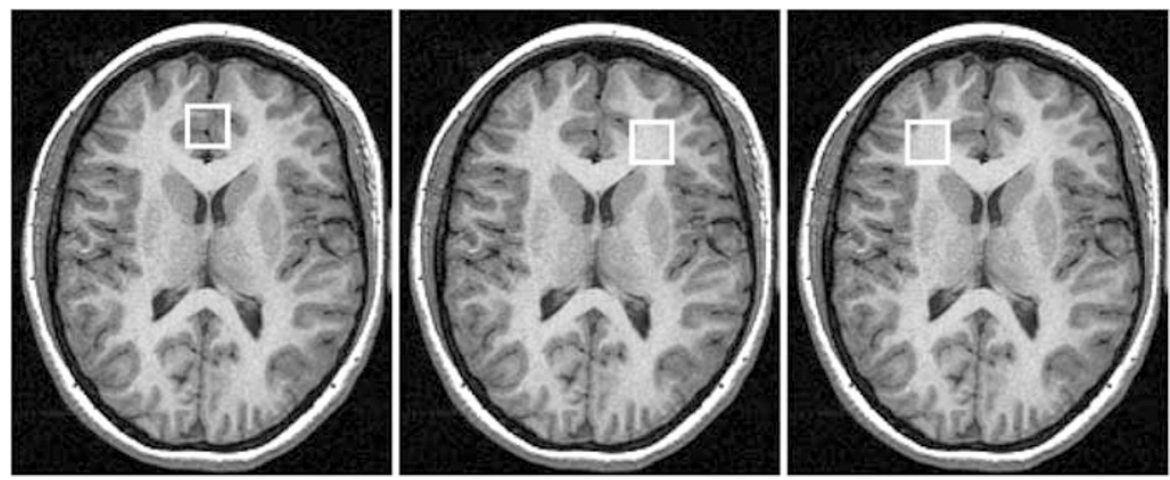

Figure I Placement of medial, and left and right lateral ventral prefrontal voxels.

reported with a confidence measurement (SD\%) reflecting maximum likelihood estimates and their uncertainties (Cramer-Rao lower bounds) (Provencher, 2001). Metabolite concentrations with an SD\% of $<10$ were included in the study. Using this criterion, one patient had unusable data in the left ventral prefrontal cortex at baseline and therefore, this patient was not included in any analyses of left ventral prefrontal metabolites. Two other patients did not have usable data in the right ventral prefrontal cortex at day 7 and therefore, these patients were only included in analyses of right ventral prefrontal metabolites that examined baseline predictors of remission.

Gray and white matter and cerebral spinal fluid(CSF) contribution to voxel volumes were determined using a $\mathrm{K}$-means segmentation algorithm employed for analysis of the FSPGR sequence incorporated into custom software (Table 1). Metabolite concentrations were adjusted for amount of CSF in each voxel (Cecil et al, 2002). Specifically, the LC Model software determines the metabolite concentrations assuming a parenchymal volume as prescribed $(8 \mathrm{cc})$. However, particularly in the medial prefrontal gray matter voxel, CSF contributions within the gyral sulcus were approximately $15 \%$ of the total volume. There were negligible CSF levels in the white matter voxels (see Table 1). To compensate for the parenchymal assumption, we used custom software to determine gray, white, and CSF contributions to each voxel for each subject for each scan. We then applied a scale factor based upon the ratio of volume of CSF to total volume and adjusted each metabolite concentration accordingly.

\section{Statistical Analyses}

Demographics and clinical characteristics of bipolar and comparison subjects are listed in Table 2. All HC adolescents $(N=10)$ underwent three MRS scans at the same time points, and were included in analyses. Within the bipolar group, one patient failed to return for any follow-up. Therefore, only 19 adolescents with bipolar disorder were included in the intent-to-treat (ITT) sample and in the clinical outcome analyses. Demographic variables were identified as potential covariates for group differences between bipolar and $\mathrm{HC}$ adolescents, as well as between bipolar adolescents who were remitters and those who were nonremitters using $t$-tests or Fisher's Exact tests and a liberal $p$-value of 0.2 for differences between groups.
Table I Percent of Gray (GM), White Matter (WM), and Cerebral Spinal Fluid (CSF) in Medial and Left and Right Lateral Ventral Prefrontal Voxels of Bipolar $(N=19)$ and Healthy Comparison $(N=10)$ Subjects

\begin{tabular}{lccc}
\hline Region of interest & $\begin{array}{c}\text { BP remitters } \\
(\mathbf{N}=\mathbf{I} \mathbf{)})\end{array}$ & $\begin{array}{c}\text { BP } \\
\text { nonremitters } \\
\mathbf{( N = 8 )}\end{array}$ & $\begin{array}{c}\text { HC } \\
(\mathbf{N}=\mathbf{I 0})\end{array}$ \\
\hline $\begin{array}{l}\text { Medial VPFC, mean (SD) } \\
\text { \% GM }\end{array}$ & $65(4)$ & $63(5)$ & $67(5)$ \\
\% CSF & $16(3)$ & $16(2)$ & $14(5)$ \\
& & & \\
Left lateral VPFC, mean (SD) & $82(6)$ & $83(5)$ & $85(6)$ \\
\% WM & $0.9(0.8)$ & $0.7(0.6)$ & $0.5(0.7)$ \\
\% CSF & & & \\
Right lateral VPFC, mean (SD) & $85(6)$ & $82(5)$ & $87(6)$ \\
\% WM & $0.5(0.5)$ & $0.8(0.9)$ & $0.4(0.4)$ \\
\% CSF & & & \\
\hline
\end{tabular}

$\mathrm{VPFC}=$ ventral prefrontal cortex; $\mathrm{BP}=$ bipolar subjects; $\mathrm{HC}=$ healthy comparison subjects.

Analyses of variance (ANOVAs) were utilized to calculate change in total YMRS and CDRS-R scores over time, using the last observation carried forward (LOCF) from the ITT sample of bipolar adolescents $(N=19)$. Response was defined by $a \geqslant 50 \%$ reduction in YMRS score from baseline to end point. Remission was based on previously published criteria and defined by (1) an end point YMRS score of $\leqslant 12$, (2) an end point CDRS-R $\leqslant 40$, (3) an end point CGIBP-I score of 1 or 2 (much or very much improved), and (4) an end point CGAS score $\geqslant 51$ (Pavuluri et al, 2005; Findling et al, 2005). Paired $t$-tests were used to compare change from baseline to end point in rating scale scores between remitters and nonremitters. Individual adverse events were summarized by frequency. Paired $t$-tests were also used to compare change from baseline to end point in laboratory measures, EPS rating scale scores, and vital signs.

Within the each group (HC and bipolar adolescents), change over time in NAA concentrations in each of the three voxels of interest (medial, left, and right prefrontal 
Table 2 Comparison of Demographic and Clinical Variables Among First-Hospitalization Manic Bipolar Adolescents who were Olanzapine Remitters $(N=\mid \mathrm{I})$ and Nonremitters $(N=8)$ and Healthy Comparison Subjects $(N=10)$

\begin{tabular}{|c|c|c|c|}
\hline Variable & $\begin{array}{c}\text { BP remitters } \\
(N=I I)\end{array}$ & $\begin{array}{c}\text { BP } \\
\text { nonremitters } \\
(\mathbf{N}=\mathbf{8})\end{array}$ & $\begin{array}{c}\mathrm{HC} \\
(N=10)\end{array}$ \\
\hline Age, mean (SD) & $14(2)$ & $15(2)$ & $15(2)$ \\
\hline Sex, females, $N(\%)$ & $7(64)$ & $6(75)$ & $6(60)$ \\
\hline Race, Caucasian, N (\%) & $10(9 \mid)$ & $6(75)$ & $8(80)$ \\
\hline Tanner stage & $3.8(1.1)$ & $3.8(1.2)$ & $3.6(1.0)$ \\
\hline ADHD, N (\%) & $5(45)$ & $3(38)$ & N/A \\
\hline Psychotic features, N (\%) & $5(45)$ & $2(25)$ & N/A \\
\hline YMRS, mean (SD) & & & N/A \\
\hline Baseline & $33(7)$ & $34(6)$ & \\
\hline End point ${ }^{\mathrm{a}}$ & $6(2)$ & $23(9)$ & \\
\hline CDRS-R, mean (SD) & & & $N / A$ \\
\hline Baseline & $5 \mid(17)$ & $53(22)$ & \\
\hline End point & $25(6)$ & $37(16)$ & \\
\hline CGAS, mean (SD) & & & N/A \\
\hline Baseline & $33(6)$ & $30(9)$ & \\
\hline End point ${ }^{b}$ & $7 \mid(10)$ & $52(7)$ & \\
\hline
\end{tabular}

${ }^{a} t=5.5, p=0.0006$, nonremitters $>$ remitters.

$b_{t}=2.3, p=0.001$, remiiters $>$ nonremitters.

$\mathrm{BP}=$ bipolar subjects; $\mathrm{HC}=$ healthy comparison subjects; $\mathrm{ADHD}=$ attention deficity hyperactivity disorder; YMRS = Young Mania Rating Scale; CDRS$\mathrm{R}=$ Children's Depression Rating Scale, revised; CGAS = Children's Global Assessment Scale.

cortex) was evaluated using repeated measures ANOVA (PROC MIXED). Post hoc $t$-tests were performed to identify the time period during which any significant change in NAA occurred. We then divided the bipolar adolescent sample into remitters and nonremitters, in order to examine the effects of categorical symptom resolution on MRS measures. Change in NAA from baseline to day 28 within each voxel of interest for each individual was calculated and the mean change was compared between bipolar remitters and nonremitters, as well as within each group, using $t$-tests.

Pearson correlations were performed to identify statistically significant associations among change in NAA concentrations and clinical variables, including change in YMRS and CDRS-R scores.

As secondary analyses, changes in other metabolite concentrations were also examined using repeated measures ANOVAs (PROC MIXED), Bonferroni corrected for multiple comparisons $(p<0.004)$. Additionally, differences in baseline metabolite concentrations between remitters and nonremitters were evaluated using $t$-tests, Bonferroni corrected for multiple comparisons $(p<0.003)$. All analyses were performed using the Statistical Analysis System (SAS) for the PC (SAS Institute, Cary, NC, 1999). Other analyses were performed as necessary.

\section{RESULTS}

\section{Baseline Characteristics of Bipolar and Healthy Comparison Adolescents}

Of the 19 bipolar adolescents, 18 (95\%) completed the 4-week trial of olanzapine. One patient discontinued prematurely (at day 18) due to lack of efficacy. All of the healthy comparison subjects $(N=10)$ completed MRS scans at baseline and on days 7 and 28 . There were no significant group differences in age, sex, race, or Tanner stage between bipolar and comparison adolescents (Table 2). The mean (SD) dosage of olanzapine at day 5 until end point was 12.7 (4.8) $\mathrm{mg} /$ day.

\section{Efficacy Measures}

A repeated measures ANOVA revealed a statistically significant reduction over time in YMRS score (baseline, mean $(\mathrm{SD})=33.6(6.6)$, end point, mean $(\mathrm{SD})=13.0(10.3)$, $\mathrm{F}(3,52)=26.2, p<0.0001)$ (Figure 2). In total, 74\% (14/19) of the bipolar adolescents were YMRS responders. A total of $59 \%(11 / 19)$ of the bipolar adolescents were remitters (end point $\mathrm{YMRS} \leqslant 12$, CDRS-R $\leqslant 40$, CGI-I $\leqslant 2$, and CGAS $\geqslant 51$ ) following treatment with olanzapine. CDRS-R scores were significantly reduced and CGAS score significantly increased from baseline to end point (baseline CDRS- $R$, mean $(\mathrm{SD})=52.3$ (18.5), end point CDRS-R, mean $(\mathrm{SD})=29.7$ (12.4), $\mathrm{F}(3,52)=12.2, p<0.0001$ ) (baseline CGAS, mean $(\mathrm{SD})=31.8(7.2)$, end point CGAS, mean $(\mathrm{SD})=63.2(12.8)$, $t=9.8, p<0.0001)$. There were no statistically significant differences in demographic or baseline clinical variables between bipolar adolescents who were and were not remitters (Table 2).

\section{Tolerability and Side Effects}

There were no significant changes from baseline to end point in glucose level, QTc interval, TSH, white blood count (WBC), hematocrit, platelet count, EPS ratings, or LFTs. Mean (SD) random serum glucose level at baseline was 85 (3) and at end point was 81 (4). There were no occurrences of orthostatic hypotension. There was a statistically and

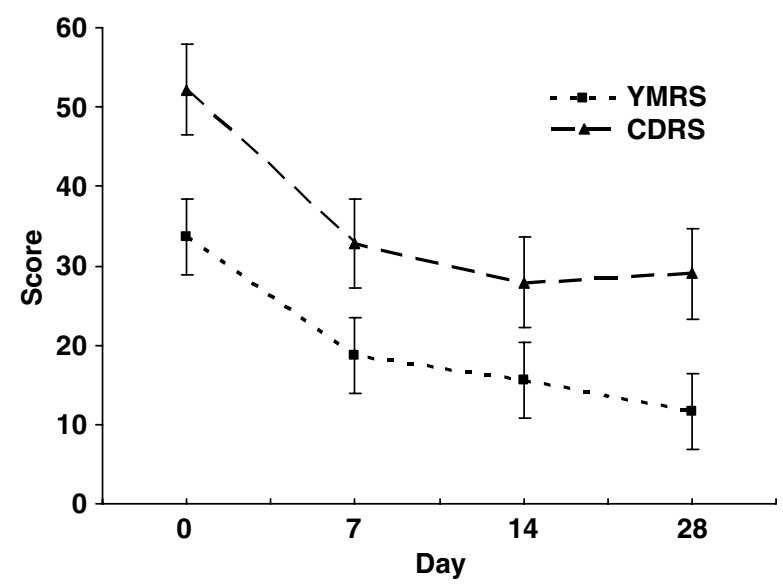

Figure 2 Change in YMRS $(p<0.000 \mathrm{I})$ and CDRS $(p<0.000 \mathrm{I})$ scores over 28 days in manic BP adolescents $(N=19)$ treated with olanzapine. 
clinically significant increase in weight from baseline to end point (weight gain, mean $(\mathrm{SD})=5.3(3.6) \mathrm{kg}, t=10.3$, $p<0.0001)$.

There were no serious adverse events. The most common adverse events were sedation $(13 / 19,68 \%)$, nausea $(7 / 19$, $37 \%)$, and increased appetite $(6 / 19,32 \%)$. Sedation was more frequent in nonremitters $(8 / 8,100 \%)$ than in remitters $(5 / 11,45 \%$, Fisher's exact test, $p=0.04)$. All side effects were rated as mild to moderate by the subjects and their caregivers.

\section{${ }^{1} \mathrm{H}$ MRS}

There was no statistically significant difference in percentages of gray matter, white matter, or cerebrospinal fluid in the medial, nor left or right lateral prefrontal voxels among healthy controls, bipolar remitters, and bipolar nonremitters (Table 1). Nonetheless, metabolite concentrations were adjusted for amount of CSF in each voxel (Cecil et al, 2003). Within the $\mathrm{HC}$ adolescents there were no statistically significant changes over time in NAA, Cho, Cr, mI, Glx in any of the voxels studied (all $p>0.1$ ) (Table 3 ).

Bipolar adolescents did not exhibit a significant change over time in NAA in any of the three prefrontal voxels $(p>0.2)$. However, there was a statistically significant difference in change over time in medial prefrontal NAA between olanzapine remitters and nonremitters $(t=3.2$, $p=0.006)$. Specifically, from baseline to end point, NAA levels decreased in nonremitters $(t=-2.9, p=0.03)$ and increased in remitters $(t=2.4, p=0.05)$ (Figure 3$)$. Moreover, within the bipolar adolescents change from baseline to end point in medial prefrontal NAA was positively associated with decrease from baseline to end point in YMRS score $(r=0.68, p=0.004)$ (Figure 4).

Bipolar adolescents had a statistically significant change over time in medial prefrontal choline level $(\mathrm{F}(2,28)=15.7$, $p=0.0001)$. Specifically, there was a statistically significant increase in choline concentration from baseline to day 7 $(t=-3.7, p=0.002)$. Bipolar adolescents also exhibited a change over time in right lateral prefrontal choline level $(\mathrm{F}(2,26)=5.3, p=0.02)$, although after correcting for multiple comparisons this finding was no longer statistically significant. Specifically, there was an increase in right lateral prefrontal choline concentration from baseline to day 7 $(t=-2.3, p=0.02)$. There were no statistically significant differences in change from baseline to end point in medial prefrontal or right lateral prefrontal choline between remitters and nonremitters ( $p=0.8$ and 0.7 , respectively). There were no other statistically significant changes over time in metabolite concentrations within the group of bipolar adolescents. Baseline medial prefrontal choline was significantly greater in olanzapine remitters as compared to nonremitters $(t=-3.9, p=0.001)$ (Figure 5). There were no other statistically significant differences in baseline metabolite concentrations or changes over time in metabolites levels between remitters and nonremitters.

\section{DISCUSSION}

The results of our study suggest that manic bipolar adolescents exhibit high response (74\%) and remission
Table 3 Metabolite Concentrations in the Ventral Medial Prefrontal Voxel at Baseline, Day 7 and End Point in Bipolar $(N=19)$ and Healthy $(N=10)$ Adolescents

\begin{tabular}{|c|c|c|c|}
\hline Metabolite (mM) & $\begin{array}{c}\text { BP remitters } \\
(N=I I)\end{array}$ & $\begin{array}{c}\text { BP } \\
\text { nonremitters } \\
(N=8)\end{array}$ & $\begin{array}{c}\mathrm{HC} \\
(N=10)\end{array}$ \\
\hline \multicolumn{4}{|l|}{ NAA, mean (SD) } \\
\hline Baseline & $7.8(0.5)$ & $7.9(0.4)$ & $7.7(0.3)$ \\
\hline Day 7 & $7.8(0.5)$ & $8.1(0.5)$ & $7.9(0.5)$ \\
\hline End point & $8.2(0.8)$ & $7.5(0.5)$ & $7.8(0.3)$ \\
\hline \multicolumn{4}{|c|}{ Myo-inositol, mean (SD) } \\
\hline Baseline & $4.0(0.4)$ & $3.9(0.4)$ & $4.3(0.4)$ \\
\hline Day 7 & $4.0(0.6)$ & $3.7(0.9)$ & $4.0(0.3)$ \\
\hline End point & $4.4(0.3)$ & $3.9(0.6)$ & $4.3(0.3)$ \\
\hline \multicolumn{4}{|l|}{ Choline, mean (SD) } \\
\hline Baseline & $1.2(0.09)$ & $1.0(0.1)$ & $1.3(0.2)$ \\
\hline Day 7 & $1.4(0.1)$ & $1.3(0.2)$ & $1.2(0.2)$ \\
\hline End point & $1.4(0.1)$ & $1.2(0.1)$ & $1.2(0.2)$ \\
\hline \multicolumn{4}{|l|}{ Creatine, mean (SD) } \\
\hline Baseline & $5.4(0.4)$ & $5.4(0.3)$ & $5.7(0.4)$ \\
\hline Day 7 & $5.5(0.4)$ & $5.7(0.4)$ & $5.7(0.6)$ \\
\hline End point & $5.7(0.4)$ & $5.4(0.6)$ & $5.6(0.6)$ \\
\hline \multicolumn{4}{|l|}{ Glx, mean (SD) } \\
\hline Baseline & |3.2(1.6) & $12.6(1.5)$ & $12.0(1.0)$ \\
\hline Day 7 & $12.1(1.4)$ & |3.1(1.1) & $12.7(0.9)$ \\
\hline End point & |3.3(1.1) & $12.5(1.4)$ & $12.6(1.2)$ \\
\hline
\end{tabular}

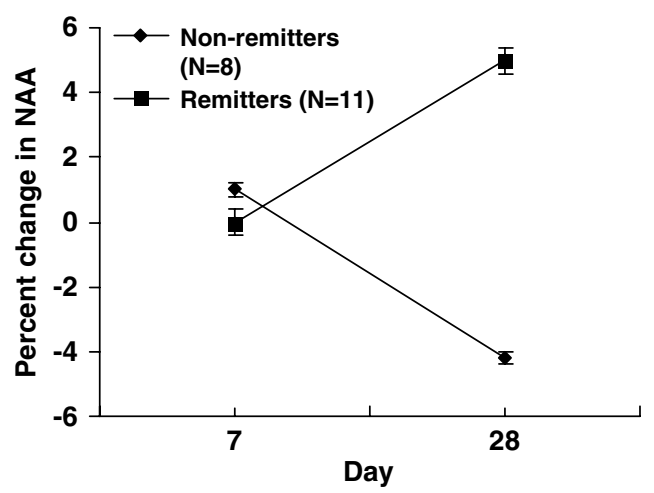

Figure 3 Percent change in medial prefrontal N-acetyl-aspartate(NAA) concentrations 7 and 28 days following treatment with olanzapine between first-hospitalization manic adolescents who were remitters $(N=I \mathrm{I})$ and nonremitters $(N=8)^{*}, \quad(F(I, \mid 6)=9.8, p=0.007)$. *One nonremitter discontinued treatment and received their end point MRS scan on day 14 , but was included in the day 28 data.

(59\%) rates following treatment with olanzapine. Furthermore, olanzapine significantly reduces both symptoms of mania and depression associated with adolescent bipolar disorder. Although most of the patients in our study experienced significant weight gain, olanzapine was well 


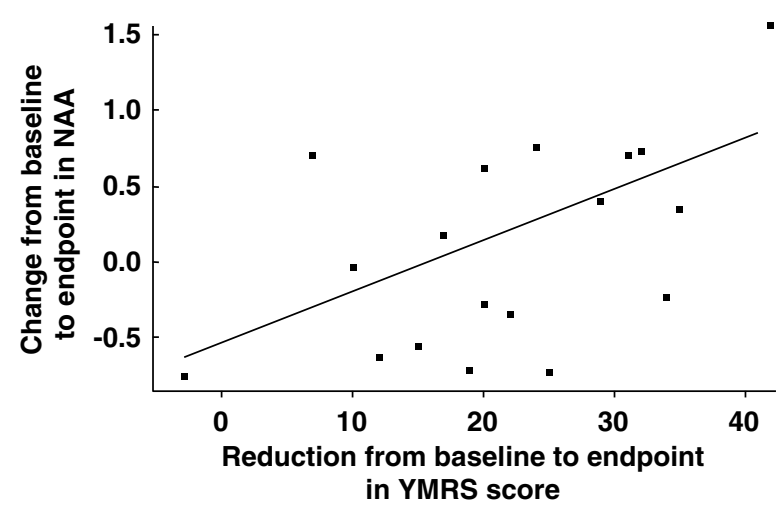

Figure 4 Association between reduction from baseline to end point in YMRS score and change from baseline to end point in medial prefrontal NAA concentration in first-hospitalization manic adolescents $(N=19)$ treated with olanzapine $(r=0.68, p=0.004)$.

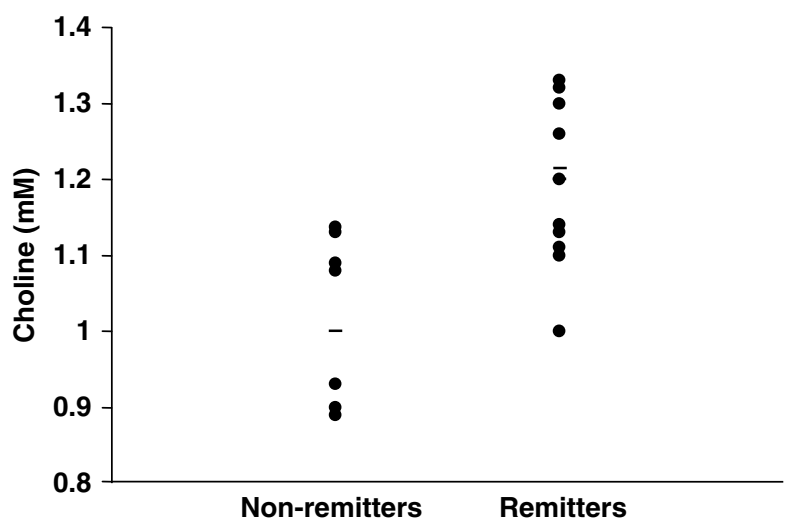

Figure 5 Baseline medial ventral prefrontal choline levels in firsthospitalization manic adolescents who were nonremitters $(N=8)$ and remitters $(N=I I)$ to olanzapine treatment $(p=0.00 I)$.

tolerated with few other side adverse effects. Our results are consistent with the findings of other case and open-label reports of olanzapine in bipolar children and adolescents (Frazier et al, 2001; Chang and Ketter, 2000; Soutullo et al, 1999). However, in order to establish the efficacy of olanzapine in manic youth, double-blind placebo-controlled studies are needed.

Our findings indicate that bipolar manic adolescents who were successfully treated with olanzapine have an increase in medial prefrontal NAA levels, suggesting that remission following olanzapine treatment is associated with neurotrophic effects, as suggested in the introduction (Parikh et al, 2004a,b). Specifically, increases in NAA following successful treatment of mania with olanzapine may lead to increased MVPF neuronal viability and/or function as compared to unsuccessful treatment with olanzapine. Although the reason for finding NAA changes only in the MVPF voxel is unclear, this voxel had the greatest amount of gray matter, which may be more sensitive to NAA changes, and was the only voxel to include the anterior cingulate and orbital frontal cortex, both of which are thought to be involved in the neurophysiology of mood regulation (Strakowski et al, 2005). Healthy controls did not demonstrate any significant difference in any metabolite over the 28 days of this study, suggesting that without the effects of medication there is stability and reproducibility in our measurements of metabolite concentrations. However, since healthy controls did not receive olanzapine, whether the increase in NAA is secondary to the reduction in manic or depressive symptoms, that is, indirectly related to olanzapine therapy, or directly to olanzapine exposure remains unclear. We observed an increase in medial prefrontal NAA primarily between days 7 and 28 in olanzapine remitters. Additionally, within the bipolar adolescents (remitters and nonremitters), increases in NAA were significantly associated with decreases in manic symptoms, suggesting that NAA increases are related to resolution of manic symptoms rather than a direct effect of olanzapine treatment. However, not all medications that successfully treat mania have similar effects on prefrontal NAA concentrations. As noted previously, although lithium has been associated with increases in prefrontal NAA levels (Moore et al, 2000b), valproate has been associated with decreases in NAA levels in adults with bipolar disorder (Silverstone et al, 2003; Cecil et al, 2002).

Several MRS studies indicate abnormal NAA concentrations in the brains of bipolar adolescents compared with healthy subjects (Cecil et al, 2003; Chang et al, 2003). More recently, Geller et al (2004) identified that the BDNF Val66 allele confers susceptibility to a prepubertal and early adolescent bipolar disorder phenotype. Additionally, the BDNF Met66 substitution has been associated with lower hippocampal NAA in healthy subjects (Egan et al, 2003) and a therapeutic response to atypical antipsychotics in patients with schizophrenia (Hong et al, 2003). In another study, cognitive functioning was significantly better in subjects with $\mathrm{Val} / \mathrm{Val}$ BDNF genotype compared with $\mathrm{Val} / \mathrm{Met}$ genotype (Rybakowski et al, 2003). Future studies examining whether the association between olanzapine-induced remission and increases in prefrontal NAA is specific to bipolar youth and whether bipolar youth with the Val66 or Met66 BDNF gene are more likely to respond to olanzapine, might clarify the meaning of these associations.

Alternatively, the observed increase in NAA may indicate a correction of abnormal mitochondrial metabolism. Based on recent findings of elevated lactate and glutamine/ glutamate/ $\gamma$-aminobutyric acid (GABA) in the frontal gray matter of unmedicated bipolar adults and decreased purine levels following choline administration (Lyoo et al, 2003), investigators have hypothesized that mitochondrial abnormalities may be present in bipolar patients (Dager et al, 2004). NAA is reduced by mitochondrial respiratory chain inhibitors (Bates et al, 1996), and therefore, increases in NAA may indicate correction of impaired mitochondrial energy production in olanzapine remitters.

Our findings also indicate that higher levels of baseline medial prefrontal choline predicted olanzapine-induced remission. Moreover, bipolar adolescents, regardless of remission status, experienced an increase in medial prefrontal gray and right lateral prefrontal white matter choline within the initial week of olanzapine treatment, suggesting that an increase in choline may be an immediate and direct biochemical effect of olanzapine, rather than the result of resolution of manic symptoms. Several investigators have hypothesized involvement of cholinergic neurotransmission in the pathophysiology of bipolar disorder 
(Stoll et al, 1991; Bymaster et al, 2003). Indeed, lithium inhibits choline membrane transport and thus leads to an increase in intracellular choline. However, it is unclear whether choline transport inhibition is a direct therapeutic property of lithium or is a secondary effect of lithium treatment (Bymaster and Felder, 2002).

Choline is a precursor and metabolite of acetylcholine. Results from prior studies demonstrate that olanzapine has a high affinity for muscarinic receptors, specifically the M2 autoreceptor (Raedler et al, 2000), and increases acetylcholine release in prefrontal regions (Ichikawa et al, 2002), which may lead to increases in choline. Choline is also precursor and metabolite of membrane phospholipids, such as phosphatidylcholine. The membrane choline constitutes the bulk of the Cho peak in ${ }^{1} \mathrm{H}$ MRS, which may represent a marker for membrane phospholipid metabolism. Phosphatidylcholine is an important source of second messenger diacylglycerol. Increases in choline lead to inhibition of phosphatidylcholine hydrolysis and decreases second messenger signaling through inhibition of the phosphatidylcholine system. Indeed, abnormalities in second messenger signaling pathways have been associated with bipolar disorder (Moore et al, 1999; Brunello and Tascedda, 2003), and choline supplementation has been useful as adjunctive treatment for patients with bipolar disorder (Stoll et al, 1996). Although purely speculative, perhaps olanzapine may exert its antimanic effects by increasing choline levels, which subsequently initiates a cascade of events, possibly through inhibition of overly active second messenger systems or membrane effects, which eventually leads to symptom response, similar to the hypothesized mechanism of action of lithium (Moore et al, 1999). Furthermore, we observed that bipolar adolescents with higher baseline choline may be more likely to experience remission with olanzapine, suggesting that adolescents with higher baseline choline who are treated with olanzapine may be more likely to have enough choline to inhibit cell membrane breakdown and second messenger systems as opposed to those with lower baseline choline levels.

One previous MRS study examined medication effects or predictors of response in bipolar children and adolescents. In this study, response to lithium treatment in bipolar children and adolescents was associated with significant decreases in anterior cingulate myo-inositol concentrations. However, this study was confounded by patients receiving multiple medications. Moreover, baseline predictors of response were not identified in this study (Davanzo et al, 2001). Although the results of our study should be considered preliminary and interpreted with caution because of the limited number of patients evaluated, perhaps, in the future MRS may be utilized at the time of initial diagnosis to determine which medications will be most effective for a specific bipolar adolescent and thereby, would decrease the morbidity and mortality associated with ineffectively treated mania in youth. Obviously, an initial step towards this goal is to replicate and expand upon the findings of these investigations.

There are several limitations to this study that should be considered when interpreting the results. First, we studied bipolar adolescents at the time of their first hospitalization for a manic or mixed episode. Since most of these adolescent were relatively early in their illness, they may exhibit a greater treatment response rate and different metabolite profiles than samples of bipolar adolescents or adults who are more chronically ill. On the other hand, the findings are not confounded by prior treatment or the nonspecific effects of illness chronicity. Second, the small sample size of our study limited the power of this study to detect other differences in metabolites that might exist. Third, we used an arbitrary definition of clinical remission to divide the sample of bipolar adolescents into remitters and nonremitters. Although correlations between ratings and metabolite changes support the findings, the results may have been different had we used other definitions of remission. Fourth, from this study we are unable to determine whether our findings are specific to neurochemical effects of olanzapine in manic bipolar adolescents, olanzapine treatment in general, or resolution of mania. Future studies comparing the effects of atypical antipsychotics and mood stabilizers on choline and NAA in healthy subjects as well as in patients with bipolar and other psychiatric disorders will clarify the specificity of our results. Fifth, since we used a short echo time of $35 \mathrm{~ms}$, we cannot rule out the possibility that alterations in macromolecules may have contributed to the changes in NAA peaks. Finally, recent findings suggest the presence of neurochemical and neurofunctional abnormalities in the prefrontal cortex of bipolar youth (Cecil et al, 2003; Chang et al, 2003, 2004; Blumberg et al, 2004). We, therefore, investigated the effects of olanzapine on prefrontal metabolite concentrations. However, future studies should assess the effect of olanzapine on metabolites in other brain regions; for example, choline abnormalities have been implicated in the basal ganglia of adults with bipolar disorder (Strakowski et al, 2005).

Despite the limitations of this study, the results suggest that further investigations, of longer duration and in larger samples of bipolar patients, of patients with other psychiatric disorders, and healthy controls, clarifying the effects of olanzapine on neuronal viability, function, and metabolism, as well as on second messenger systems are necessary.

\section{ACKNOWLEDGEMENTS}

This study was supported by NIMH Grants MH63373 (Dr DelBello) and MH58170 (Dr Strakowski), and NIEHS Grant ES011261 (Dr Cecil), the Stanley Medical Research Institute, and Eli Lilly Inc.

\section{REFERENCES}

AIMS (1976). ECDEU Assessment Manual for Psychopharmacology (revised edition) US Department of Health, Education and Welfare: Rockville, Maryland.

Barnes TRE (1989). A rating scale for drug induced akathisia. $\mathrm{Br} J$ Psychiatry 154: 672-676.

Bates TE, Loesch A, Burnstock G, Clark JB (1996). Mitochondrial nitric oxide synthase: a ubiquitous regulator of oxidative phosphorylation? Biochem Biophys Res Commun 218: 40-44.

Bhangoo RK, Lowe CH, Myers FS, Treland J, Curran J, Towbin KE et al (2003). Medication use in children and adolescents treated in the community for bipolar disorder. J Child Adolesc Psychopharmacol 13: 515-522. 
Blumberg HP, Kaufman J, Martin A, Charney DS, Krystal JH, Peterson BS (2004). Significance of adolescent neurodevelopment for the neural circuitry of bipolar disorder. Ann N Y Acad Sci 1021: 376-383.

Brunello N, Tascedda F (2003). Cellular mechanisms and second messengers: relevance to the psychopharmacology of bipolar disorders. Int J Neuropsychopharmacol 6: 181-189.

Bymaster FP, Felder CC (2002). Role of the cholinergic muscarinic system in bipolar disorder and related mechanism of action of antipsychotic agents. Mol Psychiatry 7: S57-S63.

Bymaster FP, Felder CC, Tzavara E, Nomikos GG, Calligaro DO, Mckinzie DL (2003). Muscarinic mechanisms of antipsychotic atypicality. Prog Neuropsychopharmacol Biol Psychiatry 27: $1125-1143$.

Cecil KM, DelBello MP, Morey R, Strakowski SM (2002). Frontal lobe differences in bipolar disorder as determined by proton MR spectroscopy. Bipolar Disord 4: 357-365.

Cecil KM, DelBello MP, Sellars MC, Strakowski SM (2003). Proton magnetic resonance spectroscopy of the frontal lobe and cerebellar vermis in children with a mood disorder and a familial risk for bipolar disorders. J Child Adolesc Psychopharmacol 13: 545-555.

Chang K, Adleman N, Dienes K, Barnea-Goraly N, Reiss A, Ketter T (2003). Decreased $N$-acetylaspartate in children with familial bipolar disorder. Biol Psychiatry 53: 1059-1065.

Chang K, Adleman NE, Dienes K, Simeonova DI, Menon V, Reiss A (2004). Anomalous prefrontal-subcortical activation in familial pediatric bipolar disorder: a functional magnetic resonance imaging investigation. Arch Gen Psychiatry 61: 781-792.

Chang KD, Ketter TA (2000). Mood stabilizer augmentation with olanzapine in acutely manic children. J Child Adolesc Psychopharmacol 10: 45-49.

Dager SR, Friedman SD, Parow A, Demopulos C, Stoll AL, Lyoo IK et al (2004). Brain metabolic alterations in medication-free patients with bipolar disorder. Arch Gen Psychiatry 61: 450-458.

Davanzo P, Thomas MA, Yue K, Oshiro T, Belin T, Strober M et al (2001). Decreased anterior cingulate myo-inositol/creatine spectroscopy resonance with lithium treatment in children with bipolar disorder. Neuropsychopharmacology 24: 359-369.

DelBello MP, Schwiers ML, Rosenberg HL, Strakowski SM (2002). A double-blind, randomized, placebo-controlled study of quetiapine as adjunctive treatment for adolescent mania. J Am Acad Child Adolesc Psychiatry 41: 1216-1223.

Egan MF, Kojima M, Callicott JH, Goldberg TE, Kolachana BS, Bertolino A et al (2003). The BDNF val66met polymorphism affects activity-dependent secretion of BDNF and human memory and hippocampal function. Cell 112: 257-269.

Findling RL, McNamara NK, Youngstrom EA, Stansbrey R, Gracious BL, Reed MD et al (2005). Double-blind 18-month trial of lithium versus divalproex maintenance treatment in pediatric bipolar disorder. J Am Acad Child Adolesc Psychiatry 44: 409-417.

Frazier JA, Biederman J, Tohen M, Feldman PD, Jacobs TG, Toma $\mathrm{V}$ et al (2001). A prospective open-label treatment trial of olanzapine monotherapy in children and adolescents with bipolar disorder. J Child Adolesc Psychopharmacol 11: 239-250.

Fristad MA, Weller EB, Weller RA (1992). The Mania Rating Scale: can it be used in children? A preliminary report. J Am Acad Child Adolesc Psychiatry 31: 252-257.

Geller B, Badner JA, Tillman R, Christian SL, Bolhofner K, Cook Jr EH (2004). Linkage disequilibrium of the brain-derived neurotrophic factor Val66Met polymorphism in children with a prepubertal and early adolescent bipolar disorder phenotype. Am J Psychiatry 161: 1698-1700.

Geller B, Zimerman B, Williams M, Bolhofner K, Craney JL, DelBello MP et al (2001). Reliability of the Washington University in St. Louis Kiddie Schedule for Affective Disorders and Schizophrenia (WASH-U-KSADS) mania and rapid cycling sections. J Am Acad Child Adolesc Psychiatry 40: 450-455.

Hong CJ, Yu YW, Lin CH, Tsai SJ (2003). An association study of a brain-derived neurotrophic factor Val66Met polymorphism and clozapine response of schizophrenic patients. Neurosci Lett 349: 206-208.

Ichikawa J, Dai J, O'Laughlin IA, Fowler WL, Meltzer HY (2002). Atypical, but not typical, antipsychotic drugs increase cortical acetylcholine release without an effect in the nucleus accumbens or striatum. Neuropsychopharmacology 26: 325-339.

Lindquist DM, Hawk RM, Karson CN, Komoroski RA (2000). Effects of antipsychotic drugs on metabolite ratios in rat brain in vivo. Magn Reson Med 43: 355-358.

Lish JD, Dime-Meenan S, Whybrow PC, Price RA, Hirschfeld RM (1994). The National Depressive and Manic-depressive Association (DMDA) survey of bipolar members. J Affect Disord 31: 281-294.

Lyoo IK, Demopulos CM, Hirashima F, Ahn KH, Renshaw PF (2003). Oral choline decreases brain purine levels in lithiumtreated subjects with rapid-cycling bipolar disorder: a doubleblind trial using proton and lithium magnetic resonance spectroscopy. Bipolar Disord 5: 300-306.

McConville BJ, Arvanitis LA, Thyrum PT, Yeh C, Wilkinson LA, Chaney RO et al (2000). Pharmacokinetics, tolerability, and clinical effectiveness of quetiapine fumarate: an open-label trial in adolescents with psychotic disorders. J Clin Psychiatry 61: 252-260.

Moore GJ, Bebchuk JM, Hasanat K, Chen G, Seraji-Bozorgzad N, Wilds IB et al (2000a). Lithium increases $N$-acetyl-aspartate in the human brain: in vivo evidence in support of bcl-2's neurotrophic effects? Biol Psychiatry 48: 1-8.

Moore GJ, Bebchuk JM, Parrish JK, Faulk MW, Arfken CL, StrahlBevacqua J et al (1999). Temporal dissociation between lithiuminduced changes in frontal lobe myo-inositol and clinical response in manic-depressive illness. Am J Psychiatry 156: 1902-1908.

Moore GJ, Bebchuk JM, Wilds IB, Chen G, Manji HK, Menji HK (2000b). Lithium-induced increase in human brain grey matter. Lancet 356: 1241-1242.

Morris N (1980). Validation of a self-administered instrument to assess stage of adolescent development. J Youth Adolesc Dev 9: 271-280.

National Institute of Mental Health (1985). CGI (Clinical Global Impressions) Scale. Psychopharmacol Bull 21: 839-843.

Parikh V, Evans DR, Khan MM, Mahadik SP (2003). Nerve growth factor in never-medicated first-episode psychotic and medicated chronic schizophrenic patients: possible implications for treatment outcome. Schizophr Res 60: 117-123.

Parikh V, Khan MM, Terry A, Mahadik SP (2004a). Differential effects of typical and atypical antipsychotics on nerve growth factor and choline acetyltransferase expression in the cortex and nucleus basalis of rats. J Psychiatr Res 38: 521-529.

Parikh V, Terry AV, Khan MM, Mahadik SP (2004b). Modulation of nerve growth factor and choline acetyltransferase expression in rat hippocampus after chronic exposure to haloperidol, risperidone, and olanzapine. Psychopharmacology (Berl) 172 365-374.

Pavuluri MN, Henry DB, Carbray JA, Naylor MW, Janicak PG (2005). Divalproex sodium for pediatric mixed mania: a 6-month prospective trial. Bipolar Disord 7: 266-273.

Poznanski EO, Freeman LN, Mokros HBR (1985). Children's Depression Rating Scale-revised. Psychopharmacol Bull 21: 979-989.

Provencher SW (1993). Estimation of metabolite concentrations from localized in vivo proton NMR spectra. Magn Reson Med 30: 672-679.

Provencher SW (2001). Automatic quantitation of localized in vivo 1H spectra with LC Model. NMR Biomed 14: 260-264. 
Raedler TJ, Knable MB, Jones DW, Lafargue T, Urbina RA, Egan MF et al (2000). In vivo olanzapine occupancy of muscarinic acetylcholine receptors in patients with schizophrenia. Neuropsychopharmacology 23: 56-68.

Rybakowski JK, Borkowska A, Czerski PM, Skibinska M, Hauser J (2003). Polymorphism of the brain-derived neurotrophic factor gene and performance on a cognitive prefrontal test in bipolar patients. Bipolar Disord 5: 468-472.

Schaffer D, Gould MS, Brasic J, Ambrosini P, Fisher P, Bird H et al (1983). A Children's Global Assessment Scale. Arch Gen Psychiatry 40: 1228-1231.

Silverstone $\mathrm{PH}, \mathrm{Wu} \mathrm{RH}$, O'Donnell T, Ulrich M, Asghar SJ, Hanstock CC (2003). Chronic treatment with lithium, but not sodium valproate, increases cortical $\mathrm{N}$-acetyl-aspartate concentrations in euthymic bipolar patients. Int Clin Psychopharmacol 18: 73-79.

Simpson GM, Angus JW (1970). A rating scale for extrapyramidal side effects. Acta Psychiatr Scand Suppl 212: 11-19.
Stoll AL, Sachs GS, Cohen BM, Lafer B, Christensen JD, Renshaw PF (1996). Choline in the treatment of rapid-cycling bipolar disorder: clinical and neurochemical findings in lithium-treated patients. Biol Psychiatry 40: 382-388.

Soutullo CA, Sorter MT, Foster KD, McElroy SL, Keck PE (1999). Olanzapine in the treatment of adolescent acute mania: a report of seven cases. J Affect Disord 53: 279-283.

Stoll AL, Cohen BM, Hanin I (1991). Erythrocyte choline concentrations in psychiatric disorders. Biol Psychiatry 29: 309-321.

Strakowski SM, DelBello MP, Adler CM (2005). The functional neuroanatomy of bipolar disorder: a review of neuroimaging findings. Mol Psychiatry 10: 105-116.

Urenjak J, Williams SR, Gadian DG, Noble M (1993). Proton nuclear magnetic resonance spectroscopy unambiguously identifies different neural cell types. J Neurosci 13: 981-989.

Young RC, Biggs JT, Ziegler VE, Meyer DA (1978). A rating scale for mania: reliability, validity and sensitivity. Br J Psychiatry 133: 429-435. 